\title{
STOCHASTIC BEAMFORMING FOR COCHLEAR IMPLANT CODING
}

\author{
Robert P. Morse ${ }^{\mathrm{a}^{*}}$, Stephen D. Holmes ${ }^{\mathrm{a}}$, Boris Shulgin ${ }^{\mathrm{b}}$, Alexander Nikitin ${ }^{\mathrm{b}}$ and Nigel G. Stocks ${ }^{\mathrm{b}}$ \\ ${ }^{a}$ School of Life and Health Sciences, Aston University, Birmingham, B4 7ET, UK \\ ${ }^{\mathrm{b}}$ School of Engineering, University of Warwick, Coventry, CV4 7AL, UK
}

\begin{abstract}
Cochlear implants are prosthetic devices used to provide hearing to people who would otherwise be profoundly deaf. The deliberate addition of noise to the electrode signals could increase the amount of information transmitted, but standard cochlear implants do not replicate the noise characteristic of normal hearing because if noise is added in an uncontrolled manner with a limited number of electrodes then it will almost certainly lead to worse performance. Only if partially independent stochastic activity can be achieved in each nerve fibre can mechanisms like suprathreshold stochastic resonance be effective.

We are investigating the use of stochastic beamforming to achieve greater independence. The strategy involves presenting each electrode with a linear combination of independent Gaussian noise sources. Because the cochlea is filled with conductive salt solutions, the noise currents from the electrodes interact and the effective stimulus for each nerve fibre will therefore be a different weighted sum of the noise sources. To some extent therefore, the effective stimulus for a nerve fibre will be independent of the effective stimulus of neighbouring fibres.

For a particular patient, the electrode position and the amount of current spread are fixed. The objective is therefore to find the linear combination of noise sources that leads to the greatest independence between nerve discharges. In this theoretical study we show that it is possible to get one independent point of excitation (one null) for each electrode and that stochastic beamforming can greatly decrease the correlation between the noise exciting different regions of the cochlea.
\end{abstract}

Keywords: cochlear implant, stochastic resonance, beamforming, noise

\section{INTRODUCTION}

Cochlear implants are a medical intervention routinely used to enable many adults and children with profound deafness to regain functional hearing ${ }^{1,2}$. In many cases of profound deafness, the hair cells in the cochlea that normally convert sound into electrical signals have been severely damaged or completely destroyed. This can occur because of congenital factors, or happen later in life through many causes such as disease or the use of some essential pharmaceuticals. Frequently, the conditions that damage the hair cells do not greatly affect the nerve of hearing - the cochlear nerve. This enables the hearing of many profoundly deaf people to be partially restored by direct electrical stimulation of the cochlear nerve by a set of about 16 to 22 electrodes that are surgically implanted into the cochlea ${ }^{3}$. This is achieved by a cochlear implant, which has three parts: the set of surgically implanted electrodes, an external speech processor that is used to encode a microphone signal into a set of appropriate electrode signals, and a radio link that transmits signals from the external speech processor to the internal electrodes.

When cochlear implants were first introduced, candidates were considered only if they got no benefit from conventional hearing aids, which simply amplify sounds. Cochlear implants, however, have now been shown to substantially improve the quality of life for people with some residual hearing ${ }^{4,5}$. Even though speech processed through a cochlear implant sounds unnatural, speech comprehension in quiet can be very good because of the redundancy in speech ${ }^{6}$. But in poor

r.p.morse@aston.ac.uk; phone +44 (0)1782 721189

Noise and Fluctuations in Biological, Biophysical, and Biomedical Systems, edited by Sergey M. Bezrukov, Proc. of SPIE Vol. 6602, 66020U, (2007) · 0277-786X/07/\$18 - doi: 10.1117/12.725416 
listening conditions, the speech comprehension of cochlear implant users is severely impaired, even at noise levels that do not greatly perturb listeners with normal hearing. There is therefore a need to design better cochlear implant coding strategies to improve the naturalness of speech and speech comprehension. Expectations have also increased and many cochlear implant users would like the opportunity to enjoy music, which is less redundant than speech. To realize these expectations, cochlear implants must increase the amount of information that is represented by the spatio-temporal pattern of cochlear nerve discharges.

\subsection{The use of noise in cochlear implants}

The normal ear contains many sources of noise ${ }^{7,8}$, which may be an essential part of normal auditory coding ${ }^{9-14}$. We have previously proposed that Gaussian noise should be added to cochlear implant signals ${ }^{10,15,16}$. We have shown in physiological and computational studies that the addition of noise can theoretically result in nerve activity that more closely resembles that evoked by acoustic stimulation ${ }^{10,17}$, and, that the information transmitted by an array of nerve fibres can be enhanced by the addition of noise to a common input signa ${ }^{18}$. We have also shown that multiplicative noise, which is also present in the normal auditory system ${ }^{7,19}$, can theoretically enhance information transmission ${ }^{20}$, and it may be that the information transmission is optimized by some combination of additive and multiplicative noise. Besides theoretical studies, tentative evidence that noise may be part of normal coding comes from psychophysical experiments with cochlear implant users. Noise is thought to increase frequency discrimination ${ }^{21}$ and to enhance the sensitivity to amplitude modulation ${ }^{22,23}$. Benham and $\mathrm{Zeng}^{24}$ have also shown that noise can reduce the minimum detectable level of a harmonic in a complex tone, although it is not clear whether this effect is based on temporal or loudness cues.

The idea that noise can be useful is closely related to work on stochastic resonance, the phenomenon observed in some nonlinear systems where an increase in the input signal-to-noise ratio can lead to enhanced detection or information transmission. This effect, where noise can be beneficial, is now well established and has been demonstrated in many physiological experiments ${ }^{25-27}$. Our recent studies have extended this work to consider the effect of noise on the global information transmitted by an ensemble of neurons ${ }^{15,28}$. Under these conditions, another form of stochastic resonance, termed suprathreshold stochastic resonance (SSR), can occur leading to a much improved information transfer.

Given the arguments for restoring the naturally occurring noise in the deafened ear and the results from SR, we believe the case for the deliberate use of noise in cochlear implants is substantial. Traditionally, however, noise is regarded as detrimental, and with good reason: if noise is added in an uncontrolled manner, it will almost certainly lead to worse performance ${ }^{10,16,29}$. Only if partially independent stochastic activity can be achieved in each nerve fibre can mechanisms like SSR be effective. One of our central goals is therefore to develop ways in which noise can be introduced to achieve statistical independence across nerve populations. This is the focus of this paper.

\subsection{High-rate pulse trains}

Complete independence is impossible to achieve with electrical stimulation given that the deafened ear typically contains about 10,000 nerve fibres ${ }^{30}$ and a typical cochlear implant contains up to about 22 electrodes $^{3}$; it is therefore not possible to stimulate each remaining cochlear nerve fibre with a separate electrode. For this reason use of a $5 \mathrm{kHz}$ ("high-rate conditioner") in addition to the speech signal has been proposed to induce a stochastic response ${ }^{31}$. Based on a computational study, Rubinstein and Wilson ${ }^{31}$ claim that a $5-\mathrm{kHz}$ conditioner does indeed lead to independent nerve activity. Physiological experiments, however, have shown synchronization in response to sinusoidal stimulation up to at least $10 \mathrm{kHz}^{32,33}$ and the response to a $5-\mathrm{kHz}$ conditioner would therefore also be expected to be synchronized. This discrepancy between the computational and physiological results may be because the forward-recurrence time analysis used in the computational study was not powerful enough to detect the synchronization between the stimulus and the modelled response $\mathrm{s}^{34}$. The null hypothesis of the forward-recurrence test is that the discharges are independent: failure to reject the null hypothesis is therefore not proof of independence. Johnson and Kiang (1976) have previously used the same method to investigate the independence of spontaneous discharges in the normal ear. They simultaneously recorded from two cochlear nerve fibres for over $100 \mathrm{~s}$, with a bin width of $0.1 \mathrm{~ms}$, and were careful to note that their failure to reject the null hypothesis was merely consistent with the hypothesis of independence. In the study by 
Rubinstein et al., only $8 \mathrm{~s}$ of simulated data was used and the bin width was increased from $0.1 \mathrm{~ms}$ to $0.5 \mathrm{~ms}$ to get "useful recurrence-time histograms". With these changes, the resulting forward recurrence-time histogram was just within the set bounds so that the hypothesis of independence could not be rejected and the results have been taken to show a pseudospontaneous respons $\mathrm{e}^{35,36}$. These changes, however, would have substantially reduced the power of the statistical test, which may well have shown a lack of independence if a smaller bin size had been used - particularly given that the associated calculation of the vector strength was $0.26^{31}$, which is suggestive of synchronization. It is therefore not clear that the use of a high-rate conditioner leads to a completely desynchronized response.

Physiological evidence also suggests that the use of a high-rate conditioner might not fully exploit the remaining cochlear nerve fibres. Litvak et al. ${ }^{37}$ recorded the response of cat cochlear nerve to a $5-\mathrm{kHz}$ pulse train and found that $46 \%$ of fibres produced less than 5 spikes / s after the first $100 \mathrm{~s}$ of the stimulus, including $26 \%$ that stopped firing altogether. Also, $13 \%$ of fibres had discharge rates greater than the maximum spontaneous rate found in normal hearing. For those fibres that still responded after $100 \mathrm{~s}, 75 \%$ did not have exponential interspike intervals following the refractory period. The temporal response of the majority of cochlear nerve fibres was therefore unlike spontaneous activity in the normal ear ${ }^{38}$. Therefore, although use of a high-rate conditioner may have benefits for cochlear implantation the responses are markedly different to those in the normal ear. Moreover, the mechanisms involved are unclear and appear to be very dependant on the exact level of the stimulus ${ }^{31,37}$.

\subsection{Stochastic beamforming}

As noted earlier, the direct stimulation of each electrode by independent Gaussian noise would not be expected to lead to independent noise at each nerve fibre. Greater independence could be achieved by using a larger number of electrodes that are closer to the nerve fibres. The number of electrodes, however, is limited by manufacturing constraints and the greater risk of further damage to the cochlea with longer or larger diameter electrode arrays ${ }^{39}$; animal experiments have shown that such damage may cause further degeneration of the cochlear nerve. Similarly, electrode arrays that are closer to the nerve fibres are more likely to damage the cochlea.

An alternative strategy is to present each electrode with a linear combination of independent Gaussian noise sources (Fig. 1). Because the normal and the deafened the cochlea is filled with conductive salt solutions, the current from an electrode spreads throughout the cochlea ${ }^{40,41}$. The noise currents from the electrodes therefore interact and the effective stimulus for each nerve fibre will be a different weighted sum of the noise sources. To some extent therefore, the effective stimulus for a nerve fibre will be independent of the effective stimulus of neighbouring fibres and all the noise sources. We refer to this approach as stochastic beamforming.

This strategy is compatible with improved electrode designs. The strategy has similarities with beamforming where a spatial filter operates on the output of an array of microphones to enhance directional selectivity. The approach also has similarities with the deconvolution method for reducing current spread proposed by Townshend and White ${ }^{42}$; in this deconvolution method, the matrix that maps electrode currents to excitation level at each point of neural excitation is determined and the inverted matrix can theoretically be used to get excitation at a single place. The main difference is that the source signals for the stochastic beamforming are Gaussian processes rather than information-bearing signals and the effectiveness of the strategy will therefore be less sensitive to the matrix values. 


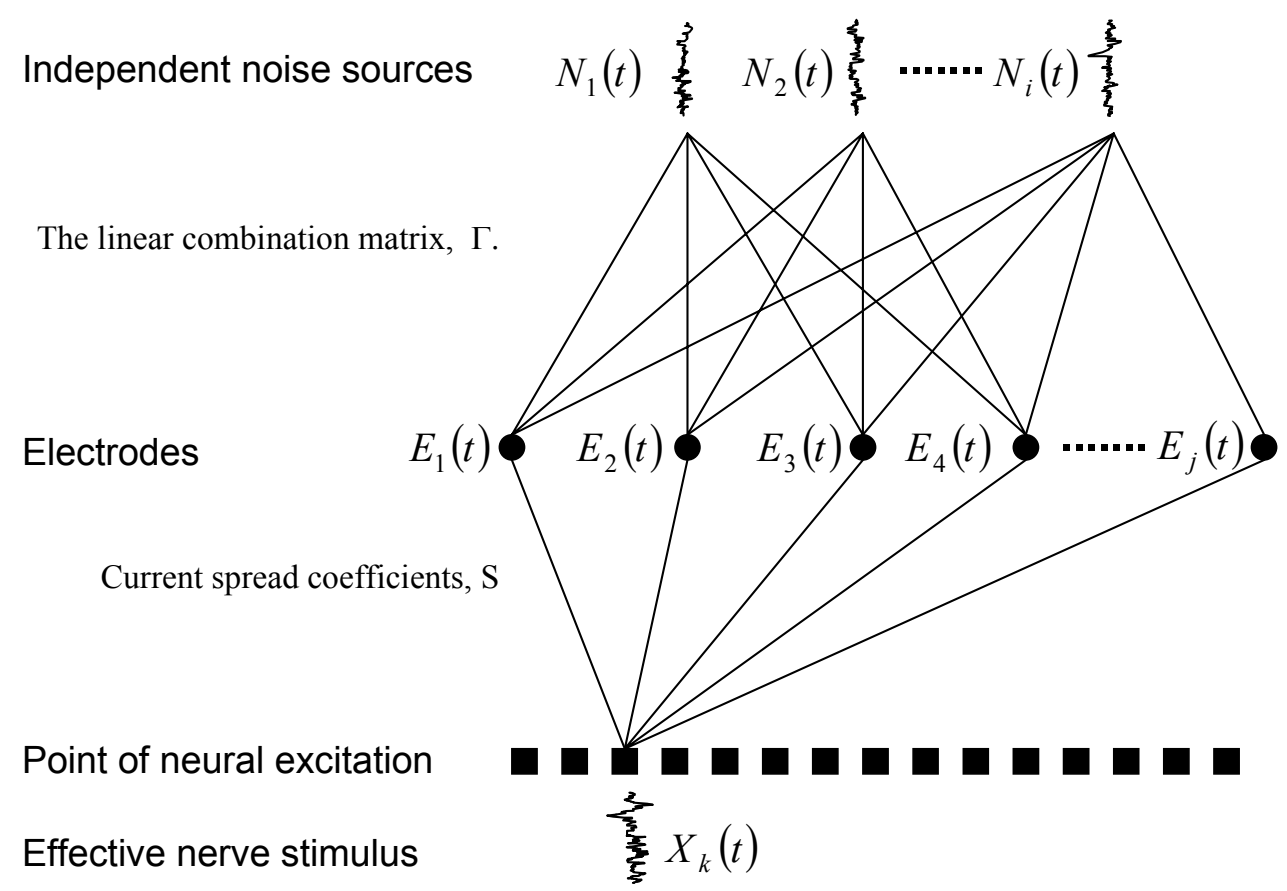

Fig.1. The stochastic beamforming strategy. Each of the $j$ electrodes is stimulated by a noise waveform, $E_{j}(t)$, that is a weighted sum of $i$ independent Gaussian noise sources; The weightings are governed by the matrix $\Gamma$. Through current spread, each of the $k$ fibres is stimulated by a different noise waveform $X_{k}(t)$, which is the weighted sum of the electrode currents; the weightings are given by the structure matrix $S$, which characterizes the distances between the fibres and the electrodes and the degree of current spread.

For a particular cochlear implant subject, the electrode position and the amount of current spread are fixed. The fundamental objective is therefore to find the linear combination of noise sources that leads to the greatest independence between nerve discharges. More specifically, with reference to Fig. 1, let $S$ be the structure matrix that represents linear current spread such that the observed signals $X$ (at specified spatial locations) are a linear combination of the electrode sources $E$ given by:

$$
X=S E
$$

The assumption that the spread of current is linear is supported by physiological measurements from the guinea pig $\operatorname{cochlea}^{43}$ and the success of lumped resistive-models of current spread ${ }^{44,45}$.

In turn let the electrode sources, $E$, be composed of a linear combination of independent noise sources, $N$, which are governed by the matrix, $\Gamma$, such that

$$
E=\Gamma N
$$

Hence the observed signals are given by

$$
X=S \Gamma N \text {. }
$$

We require that all elements of $X$ are statistically independent. This requires that the covariance matrix of $X$ is diagonal. From Equation 3, the covariance matrix of $\mathrm{X}$ can be written as

$$
<X X^{T}>=<S \Gamma N N^{T} \Gamma^{T} S^{T}>.
$$


Noting that $\left\langle N N^{T}\right\rangle=\sigma^{2} I$, (because the noise sources are independent) where $\sigma^{2}$ is the variance of the noise sources, this simplifies to:

$$
<X X^{T}>=\sigma^{2} S \Gamma \Gamma^{T} S^{T}
$$

We define a matrix $U$ such that

$$
U=S \Gamma
$$

Equation 5 can then be re-written as

$$
<X X^{T}>=\sigma^{2} U U^{T} .
$$

If follows that the covariance matrix will be diagonal if the rows of $U$ are independent (orthogonal), in which case $U U^{T}=$ $k^{2} I$, and hence

$$
<X X^{T}>=\sigma^{2} k^{2} I .
$$

From Equation 6 the required linear combination of independent noise sources is therefore given by

$$
\Gamma=S^{-1} U
$$

The analysis shows that it is possible to get one independent point of excitation (one null) for each electrode. The matrix $U$, however, is not unique and any set of orthogonal vectors can be used in its construction. The simplest matrix that diagonalises Equation 7 is the identity matrix. i.e. $U=I$, and hence, trivially, $\Gamma=S^{1}$. This enables further constraints to be placed on $U$, such as power consumption by the speech processor or the distribution of spontaneous rate across the neural array is maximally flat.

We have done a preliminary computational study on stochastic beamforming ${ }^{16}$. In this study, independent noise was presented to each electrode but the noise waveforms did not satisfy Equation 9 and the strategy was therefore unlikely to have been optimal. Nonetheless, we showed that some independence between the noise exciting different regions of the cochlea was theoretically possible. Here we show that the trivial solution where the linear combination matrix is the inverse of the current spread matrix leads to much less correlated excitation compared with the direct additive strategy.

\section{CURRENT SPREAD MODEL}

In this preliminary study of stochastic beamforming, we evaluated the method using a simple model of electrical stimulation in which the attenuation of current with distance from an electrode was modelled by an exponential decay (with a space-constant of $3.6 \mathrm{~dB} / \mathrm{mm}$ ) ${ }^{46,47}$. For simplicity, we modelled the spiral structure of the scala tympani as an uncoiled cylinder and the array of nerve fibres was modelled as being in a plane orthogonal to the electrodes. We took the length of the uncoiled cochlea to be $34 \mathrm{~mm}^{48}$ and the maximum electrode insertion depth from the round window to be $25 \mathrm{~mm}^{49}$. Furthermore, to match the Clarion cochlear implant (Advanced Bionics Ltd), the 16 electrodes in the model were spaced $1 \mathrm{~mm}$ apart (from 10 to $25 \mathrm{~mm}$ from the round window). In typical cochlear implant patients, each electrode is about 0.5 to $1 \mathrm{~mm}$ away from the nearest afferent cell body ${ }^{50,51}$, which we have taken to be the region of initial excitation. In this study, we have been conservative and taken the distance from each electrode to the nearest afferent cell body (the receptor) to be $1 \mathrm{~mm}$.

Two noise strategies were studied. In the first strategy, each electrode was directly stimulated by an independent noise source and the noise intensity at each electrode was equal. In the second strategy we used stochastic beamforming; given the geometry and current spread we calculated the current spread coefficients and then took the linear combination matrix to be the inverse of the current spread matrix, i.e. the trivial solution of Equation 9. Again, the intensities of the independent noise sources were equal. For the two strategies, a cross-correlation coefficient was used to measure the degree of independence between the effective stimulus at a reference position (the receptor closest to the central electrode in the array, i.e. electrode 8) and the effective stimulus at more distant receptors. For each strategy, the outputs 
of the independent Gaussian noise sources were calculated at 10- $\mu$ s intervals for 2 seconds and the effective stimulus for each receptor was calculated using Equation 3.

\section{RESULTS}

With the direct additive noise strategy, the correlation between the effective stimulus at the central receptor and the effective stimulus at more distant receptors decreased gradually with distance (Fig. 2). For a receptor $1 \mathrm{~mm}$ (one electrode spacing) away from the central receptor, the cross-correlation coefficient for the effective stimuli was 0.96, and, even for a receptor $7 \mathrm{~mm}$ away, the cross-correlation coefficient for the effective stimuli was 0.30 . In other words with direct additive noise, the correlation between the effective stimuli for two receptors was substantial even when the receptors were some distance apart. With stochastic beamforming, the correlation between the effective stimulus at the central receptor and the effective stimulus at more distant receptors decreased more rapidly than with direct additive strategy and was 0 when the receptor was an integer multiple of the electrode spacing away from the reference receptor. For a receptor more than $1 \mathrm{~mm}$ from the central receptor, the absolute cross-correlation coefficient for the effective stimuli was always less than 0.17 , and, when the separation between the receptors was over $3 \mathrm{~mm}$, the cross-correlation coefficient was negligible.

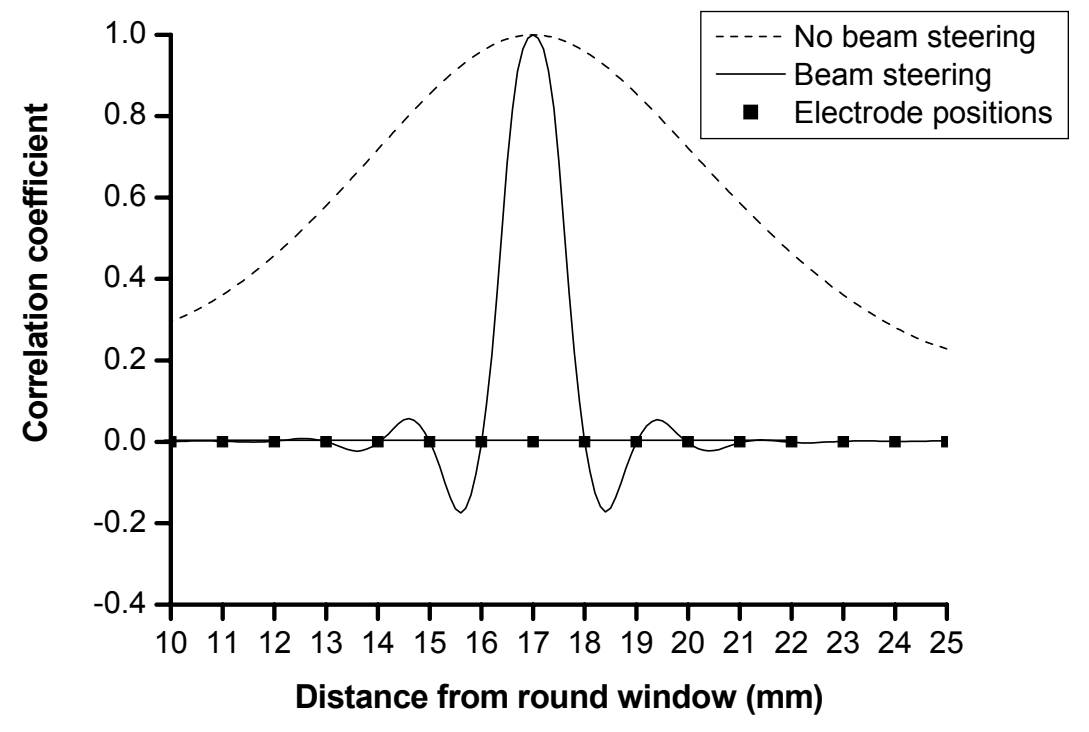

Fig.2. Correlation between the noise at a reference fibre $17 \mathrm{~mm}$ from the round window and neighbouring fibres for two noise strategies: no stochastic beamforming or beamforming. With no beaming (dashed line) each electrode was simply stimulated by an independent noise source. With beamforming, (solid line) each electrode was stimulated by a linear sum of noise sources (Equation 2). The square symbols show the positions of the electrodes within the cochlea.

\section{DISCUSSION AND CONCLUSIONS}

The simulations have demonstrated that, in principle, stochastic beamforming can be used to obtain less correlated stimulation of cochlear nerve fibres compared with a direct additive strategy. Greater information transmission through suprathreshold stochastic resonance would therefore be expected from a cochlear implant using stochastic beamforming than from one using direct additive noise.

Theoretically, the distance between the nulls is not constrained. Stochastic beamforming could therefore be used with simultaneous stimulation of all electrodes with an information-bearing waveform; in which case it may be advantageous to spread out the nulls along the cochlea. Potentially, this could optimize information transmission by maximizing the number of active fibres. Alternatively, if only one electrode transmits an information-bearing waveform at any instant 
and the signals on the electrodes are temporally interleaved, then it may be advantageous to have all the nulls in the region excited by the signal electrode; the region of nulls would then be steered to a new position each time the next electrode in sequence is stimulated. Potentially, this could optimize information transmission by maximizing the number of independent fibres. Further modeling is required to examine this trade off between the number of active fibres and their degree of independence.

The practical effectiveness of the strategy will depend on details of the current spread matrix. Because of anatomical difference between patients and various placements of the electrode array, the matrix will differ between patients. We can use one of two approaches: we can try to obtain the current-spread matrix for individual subjects and require that we get exact nulls, or we can relax the requirement for complete independence and choose the linear combination parameters to satisfy a more lenient criterion for a range of current spreads that reflects patient variability; for example, the linear combination parameters can be chosen to minimize the maximum covariance of the effective receptor stimuli over different current-spread matrices, minimize the minimum covariance of effective receptor stimuli over different current-spread matrices, or minimize the variance of the covariance values. We regard each of these options as a different stochastic beamforming strategy.

To some extent, the simulation is a conservative estimate of the benefit of stochastic beamforming. First, we have used a conservative distance of $1 \mathrm{~mm}$ between each electrode and the nearest fibre. Also, even though the cochlear nerve fibres in the deafened ear are typically not spontaneously active ${ }^{52}$, the response of cochlear nerve to electrical stimulation still has some irregularity, as demonstrated by measures of relative spread ${ }^{53}$. We have not taken the internal noise of each fibre into consideration and this noise would be expected to increase the independence of fibres for both strategies. In the simulations, however, the linear combination matrix was based on exact knowledge of the current spread matrix. In practice this matrix will have to be obtained from psychophysical experiments or measurements of the evoked compound action potential to electrical stimulation - a measurement that all modern cochlear implants can make. These measurements will not be precise and we have yet to determine the sensitivity of the method to variability in the current spread measurements.

\section{ACKNOWLEDGMENTS}

This work was funded by the EPSRC (grant GR/R35650/01 and EP/D05/1894/1(P)).

\section{REFERENCES}

1. G. M. Clark, Cochlear Implants: Fundamentals and Applications, Springer-Verlag, New York, 2003.

2. $\quad$ D. J. Allum, Cochlear implant rehabilitation in children and adults, Whurr, London, 1996.

3. S. Rosen, Cochlear implants, In Scott-Brown's Otolarngology (6th edition) Volume 2, D. Stephens, Editor, Butterworth Heinemann, Oxford. 1996.

4. B. Fraysse, N. Dillier, T. Klenzer, R. Laszig, M. Manrique, C. M. Perez, A. H. Morgan, J. MullerDeile, and A. R. Macais, "Cochlear implants for adults obtaining marginal benefits from acoustic amplification - A European study," Am. J. Otol., 19, 591-597 (1998).

5. A. Q. Summerfield and D. H. Marshall, Cochlear implantation in the UK 1990-1994: report by the MRC Institute of Hearing Research on the evaluation of the national cochlear implant program, HMSO, 1995.

6. M. F. Dorman, Speech perception by adults, In Cochlear implants: audiological foundations, R.S. Tyler, Editor, Singular Publishing Group, London. pp. 145-190. 1993.

7. E. F. Evans, Cochlear nerve and cochlear nucleus, In Handbook of sensory physiology, W.D. Keidel and W.D. Neff, Editors, Springer, Berlin. pp. 1-108. 1975.

8. H. DeVries, "Brownian motion and the transmission of energy in the cochlea," J. Acoust. Soc. Am., 24, 527-533 (1952).

9. A. Longtin, A. R. Bulsara, and F. Moss, "Time interval sequences in bistable systems and the noise-induced transmission of information by sensory neurons," Phys. Rev. Lett., 67, 656-659 (1991).

10. R. P. Morse and E. F. Evans, "Enhancement of vowel coding for cochlear implants by addition of noise," Nat. Med., 2, 928-932 (1996).

11. K. R. Henry, "Noise improves transfer of near-threshold, phase-locked activity of the cochlear nerve: evidence for stochastic resonance?," J. Comp. Physiol. A, 184, 577-584 (1999). 
12. S. Greenberg, Possible role of low and medium spontaneous rate fibres in the coding of waveform periodicity, In Auditory frequency selectivity, B. Moore and R. Patterson, Editors. pp. 241-248. 1986.

13. F. Jaramillo and K. Wiesenfeld, "Mechanoelectrical transduction assisted by Brownian motion: a role for noise in the auditory system," Nat. Neurosci., 1, 384-388 (1998).

14. Lowenstein, "Peripheral mechanisms of equilibrium," Brit. Med. Bull., 12, 114-118 (1956).

15. N. G. Stocks, D. Allingham, and R. P. Morse, "The application of suprathreshold stochastic resonance to cochlear implant coding," Noise Fluc. Lett., 2, L169-L181 (2002).

16. R. P. Morse and G. F. Meyer, "The practical use of noise to improve speech coding by analogue cochlear implants," Chaos Soliton Fract., 11, 1885-1894 (2000).

17. R. P. Morse and E. F. Evans, "Additive noise can enhance temporal coding in a computational model of analogue cochlear implant stimulation," Hear. Res., 133, 107-119 (1999).

18. D. Allingham, N. G. Stocks, R. P. Morse, and G. F. Meyer. Noise enhanced information transmission in a model of multichannel cochlear implantation. In SPIE Conference on Noise and Fluctuations. Gran Canaria, 2004.

19. R. Meddis, "Simulation of the mechanical to neural transduction in the auditory receptor," J. Acoust. Soc. Am., 79, 702-711 (1986).

20. R. P. Morse and N. G. Stocks. Enhanced cochlear implant coding using multiplicative noise. In SPIE Conference on Fluctuations and Noise. Austin, USA, 2005.

21. F.-G. Zeng, Q.-J. Fu, and R. P. Morse, "Human hearing enhanced by noise," Brain Res., 869, 251-255 (2000).

22. M. Chatterjee and M. E. Robert, "Noise enhances modulation sensitivity in cochlear implant listeners: Stochastic resonance in a prosthetic sensory system?," J. Assoc. Res. Otolarngol, 2, 159-171 (2001).

23. M. Chatterjee and S. I. Oba, "Noise improves modulation detection by cochlear implant listeners at moderate carrier levels," J. Acoust. Soc. Am., 118, 993-1002 (2005).

24. S. E. Benham and F.-G. Zeng, "Noise improves suprathreshold discrimination in cochlear implant listeners," Hear. Res., 186, 91-93 (2003).

25. J. Douglass, K. Wilkens, E. Pantazelou, and F. Moss, "Noise enhancement of information transfer in crayfish mechanoreceptors by stochastic resonance," Nature, 365, 337-340 (1993).

26. J. E. Levin and J. P. Miller, "Broadband neural encoding in the cricket cercal sensory system enhanced by stochastic resonance," Nature, 380, 165-168 (1996).

27. D. F. Russell, L. A. Wilkens, and F. Moss, "Use of behavioural stochastic resonance by paddle fish for feeding," Nature, 402, 291-294 (1999).

28. N. G. Stocks, "Suprathreshold stochastic resonance in multilevel threshold systems," Phys. Rev. Lett., 84, 2310$2313(2000)$.

29. $\quad$ B. S. Wilson, "The future of cochlear implants, "Brit. J. Audiol., 31, 205-225 (1997).

30. J. Otte, H. F. Schuknecht, and A. G. Kerr, "Ganglion cell populations in normal and pathological human cochlae. Implications for cochlear implantation," Laryngoscope, 8, 1231-1246 (1978).

31. J. T. Rubinstein, B. S. Wilson, C. C. Finley, and P. Abbas, "Pseudospontaneous activity: stochastic independence of auditory nerve fibres with electrical stimulation," Hear. Res., 127, 108-118 (1999).

32. R. Hartmann and R. Klinke, Response patterns of nerve fibres to patterned electrical stimulation, In Cochlear implants: models of the electrically stimulated ear, J.J. Miller and F.A. Spelman, Editors, Springer, New York. pp. 135-160. 1990.

33. S. B. C. Dynes and B. Delgutte, "Phase-locking of auditory nerve discharges to sinusoidal electric stimulation of the cochlea," Hear. Res., 58, 79-90 (1992).

34. R. P. Morse, T. Nunn, K. Archer, P. F. Morse, and P. Boyle, "The effect of Gaussian noise on the threshold, dynamic range, and loudness of analogue cochlear implant stimuli," J. Assoc. Res. Otolarngol, 8, 42-53 (2006).

35. R. S. Hong and J. T. Rubinstein, "High-rate conditioning pulse trains in cochlear implants: Dynamic range measures with sinusoidal stimuli," J. Acoust. Soc. Am., 114, 3327-3342 (2003).

36. R. S. Hong, J. T. Rubinstein, and D. Horn, "Dynamic range enhancement for cochlear implants," Otol. Neurotol., 24, 590-595 (2003).

37. L. Litvak, B. Delgutte, and D. Eddington, "Desynchronization of electrically evoked auditory-nerve activity by high-frequency pulse trains of long duration," J. Acoust. Soc. Am., 114, 2066-2078 (2003).

38. N. Y. S. Kiang, Discharge patterns of single fibres in the cat's auditory cortex, MIT Press, Cambridge, 1965. 
39. P. A. Leake-Jones and S. J. Rebscher, Cochlear pathology with chronically implanted scala tympani electrodes, In Cochlear Prostheses: An International Symposium, C.W. Parkins and S.W. Anderson, Editors, New York Academy of Sciences, New York. pp. 203-223. 1983.

40. F. B. Simmons and T. J. Glattke, "Comparison of electrical and acoustical stimulation of the cat ear," Ann. Otol. Rhinol. Laryngol., 81, 731-738 (1972).

41. M. M. Merzenich, D. N. Schindler, and M. W. White, "Feasibility of multichannel scala tympani stimulation," Laryngoscope, 84, 1887-1893 (1974).

42. B. Townshend and R. L. White, "Reduction of electrical interaction in auditory prostheses," IEEE Trans. Bio. Eng., 34, 891-897 (1987).

43. B. M. Clopton and F. A. Spelman, "Neural mechanisms relevant to the design of an auditory prosthesis: location and electrical characteristics," Ann. Otol. Rhinol. Laryngol., 91, 9-14 (1982).

44. M. F. Suesserman and F. A. Spelman, "Lumped-parameter model for in-vivo electrical stimulation," IEEE Trans. Bio. Eng., 40, 237-245 (1993).

45. A. Zarowski, F. J. Vanpoucke, and S. Peeters. A lumped electrical model for electrode contacts and cochlear current spread: structure, identification from EFI and in vivo results. In 2003 Conference on Implantable Auditory Prostheses. Asilomar, USA, 2003.

46. B. Wilson, C. Finley, M. Zerbi, and D. T. Lawson, Speech processors for auditory prostheses: 7th Quarterly NIH Report N01-DC-2-2401, Research Triangle Institute, 1994.

47. I. C. Bruce, M. W. White, L. S. Irlicht, S. J. O'Leary, S. Dynes, E. Javel, and G. M. Clark, "A stochastic model of the electrically stimulated auditory nerve: Single-pulse response," IEEE Trans. Bio. Eng., 46, 617-629 (1999).

48. J. B. Nadol, Jr., "Comparative anatomy of the cochlea and auditory nerve in mammals," Hear. Res., 34, 253266 (1988).

49. P. C. Loizou, "Mimicking the human ear," IEEE Signal Processing Magazine, 15, 101-130 (1998).

50. R. K. Shepherd, S. Hatsushika, and G. M. Clark, "Electrical stimulation of the auditory nerve: the effect of electrode position on neural excitation," Hear. Res., 66, 108-120 (1993).

51. C. C. Finley, B. S. Wilson, and M. W. White, Models of neural responsiveness to electrical stimulation, In Cochlear implants: models of the electrically stimulated ear, J.J. Miller and F.A. Spelman, Editors, Springer, New York. 1990.

52. N. Y. S. Kiang and E. C. Moxon, "Physiological considerations in artificial stimulation of the inner ear," Ann. Otol. Rhinol. Laryngol., 81, 714-730 (1972).

53. S. B. C. Dynes, Discharge characteristics of auditory nerve fibres for pulsatile electrical stimuli, Massachusets Institute of Technology Boston, 1996. 